\title{
SURVEY OF ENERGY HARVESTING SYSTEMS FOR WIRELESS SENSOR NETWORKS IN ENVIRONMENTAL MONITORING
}

\author{
Bogdan Dziadak ${ }^{1)}$, Lukasz Makowski ${ }^{1)}$, Andrzej Michalski ${ }^{1,2)}$ \\ 1) Warsaw University of Technology, Faculty of Electrical Engineering, Koszykowa 75, 00-661 Warsaw, Poland \\ (bogdan.dziadak@ee.pw.edu.pl,.lukasz.makowski@ee.pw.edu.pl, $\$ andrzej.michalski@ee.pw.edu.pl,+48 22 234 7427) \\ 2) Military University of Technology, Institute of Electronic Systems Gen. S. Kaliskiego 2, 00-908 Warsaw, Poland
}

\begin{abstract}
Wireless Sensor Networks (WSNs) have existed for many years and had assimilated many interesting innovations. Advances in electronics, radio transceivers, processes of IC manufacturing and development of algorithms for operation of such networks now enable creating energy-efficient devices that provide practical levels of performance and a sufficient number of features. Environmental monitoring is one of the areas in which WSNs can be successfully u sed. At the same time this is a field where devices must either bring their own power reservoir, such as a battery, or scavenge energy locally from some natural phenomena. Improving the efficiency of en ergy harvesting methods reduces complexity of WSN structures. This survey is based on practical examples from the real world and provides an overview of state-of-the-art methods and techniques that are used to create energyefficient WSNs with energy harvesting.
\end{abstract}

Keywords: environmental monitoring, wireless sensor networks, energy harvesting.

(C) 2016 Polish Academy of Sciences. All rights reserved

\section{Introduction}

Wireless Sensor Networks (WSNs) are swarms of small measurement devices that employ a microcontroller and are able to communicate with each other to cooperatively constitute a superior, networked form that is not a simple sum of these single objects but a virtual organism of nodes that unite for the network's common goal. The network's objective might be, for example, monitoring some phenomena or tracking some events at an area in which WSN was deployed.

Due to their flexibility and possibility of reconfiguration, wireless sensor networks have been widely used. WSNs can be found in medical applications, such as cardiac monitoring and acquisition of ECG signals [1,2], monitoring structures, buildings [3] and industrial processes $[4,5]$. WSNs support logistic and transport processes [6, 7], as well as military actions [8]. Furthermore, energy harvesting methods are used especially in WSNs employed in environmental monitoring.

Unfortunately, there are several limitations of WSN operation that are not easy to overcome, especially in locations that might be harmful to the WSN fragile equipment. Nodes should be self-sufficient because it may be not possible to replace batteries on site. Nodes are considered to be disposable, so that they must be inexpensive and produced in mass quantities. Another problem is minimizing the negative ecological impact of disposed equipment on its environment. For that reason batteries containing lithium or cadmium should not be used. Therefore, the energy harvesting methods and battery-less operation top the agenda of current research.

The next section presents the energy harvesting methods that can be applied in WSNs designed for environmental monitoring. The second section presents WSNs and the state of the 
art in node development. The third section reviews some applications of WSNs equipped with energy harvesting capabilities and designed specifically for environmental monitoring. Finally, there are conclusions in the last part of the paper.

\section{Possible sources of energy used in the EH, assessment of their energy efficiency.}

The term Energy Harvesting (EH) covers a number of methods that use natural physical phenomena to process various forms of energy at micro levels of electricity. Electricity obtained from these sources, with proper conditioning, can power various measurement systems. There are several issues concerning EH devices, the first one is the source of energy production: either environment or human $[9,10]$.

Most of the energy present in the environment is characterized by a very high variability of the energy efficiency, starting with constant efficiency sources, such as water micro-turbines, through sources of periodically variable efficiency, e.g. photovoltaic panels, to pulse sources that transduce kinetic energy into electricity. Most commonly utilized in EH applications are the photovoltaic, piezoelectric, thermal, biochemical, and electromagnetic phenomena. The energy efficiency of the sources used in the EH is very different. For photovoltaic sources operating in open air, the capacity is estimated at approximately $15,000 \mu \mathrm{W} / \mathrm{cm}^{3}$ and rapidly decreases to about $20 \mu \mathrm{W} / \mathrm{cm}^{3}$ for indoor facilities [11]. The expected capacity levels of mechanical vibration or air flow as a source of energy (which uses the piezoelectric effect) are of the order of $300 \mu \mathrm{W} / \mathrm{cm}^{3}$, which can be very attractive in future power systems. Depending on their geometric size, electromagnetic generators can reach the power density of a few $\mu \mathrm{W} / \mathrm{cm}^{3}$. A human body can be a kinetic or thermal energy source giving the power estimated between $5 \mu \mathrm{W}$ (for the kinetic energy) to about $40 \mu \mathrm{W}$ (for the thermal energy) [12]. Table 1 compares different energy harvesting methods [13-15].

Table 1. The power density in different energy harvesting methods.

\begin{tabular}{|c|c|c|}
\hline $\begin{array}{c}\text { Energy harvesting } \\
\text { methods }\end{array}$ & Energy harvesting elements & Power density \\
\hline $\begin{array}{c}\text { Solar and light } \\
\text { energy harvesting }\end{array}$ & photovoltaic solar panel & $1500 \mu \mathrm{W} / \mathrm{cm}^{2}$ (outdoor sun light 1000W/m2) \\
\hline Mechanical \\
energy harvesting & wind turbine & $3.5 \mathrm{~mW} / \mathrm{cm}^{2}$ \\
& $\begin{array}{c}\text { vibration piezoelectric wafer } \\
\text { vibration electromagnetic } \\
\text { vibration electrostatic }\end{array}$ & $500 \mu \mathrm{W} / \mathrm{cm}^{2}$ \\
\hline Termoelectric & termoelectric converter & $3.0 \mu \mathrm{W} / \mathrm{cm}^{2}$ \\
energy harvesting & & $3.8 \mu \mathrm{W} / \mathrm{cm}^{2}$ \\
\hline Electromagnetic wave & RF harvesting board & $40 \mathrm{~W} / \mathrm{cm}^{2}$ \\
energy harvesting & & $100 \mu \mathrm{W} / \mathrm{cm}^{2}($ temperature difference $5 \circ \mathrm{C})$ \\
\hline
\end{tabular}

When designing a wireless network of sensors, one of the most important issues is ensuring the energetic self-sufficiency of a node. There is no universal source of EH which should be selected depending on the existing environmental conditions and on the energy needs of a specific node. The energy demand of a typical WSN node used for environmental measurements depends on its operation mode. In the standby mode a typical energy demand is estimated to be up to a dozen $\mu \mathrm{W}$, whereas in the measurement mode the demand can be either small or very large - from $0.1 \mu \mathrm{W}$ to $100 \mathrm{~mW}$ during communication. These values of energy can be supplied by an efficient power supply in a sensor node based only on EH [16].

Figure 2.1 shows a universal power supply structure using EH sources. 


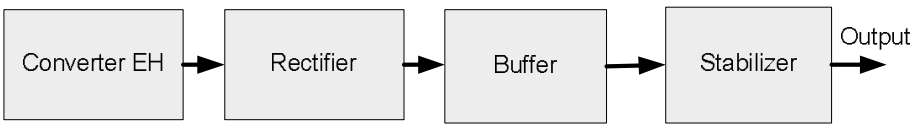

Fig. 2.1. A structure of the power supply using EH sources.

The rectifier, buffer, and stabilizer systems must have a high energy efficiency. It is difficult to specify optimal system solutions since they are selected for specific applications and methods, according to which EH is used. Undoubtedly, the most important elements in the optimization of circuits for signal conditioning are buffers that act as elements storing electricity. Batteries and supercapacitors are used most often, as they are connected to the mass of a measuring node. Their performance and longevity depend on continuous charging and discharging small portions of energy. It is one of those areas that is the subject of continuous research. Table 2 presents the most frequently used energy storage technologies [17-20].

Table 2. Typical parameters of various energy store types.

\begin{tabular}{|c|c|c|c|}
\hline Buffer type & $\begin{array}{c}\text { Specific energy } \\
\text { Wh/kg }\end{array}$ & $\begin{array}{c}\text { Energy density } \\
\text { Wh/dm }\end{array}$ & Charging cycles \\
\hline Lead acid & 40 & $70-110$ & $200-500$ \\
\hline NiCd & 60 & 180 & 1000 \\
\hline NiMH & 80 & 240 & 500 \\
\hline Li-ion & $120-200$ & $300-400$ & 500 \\
\hline Supercapacitor & $5-12$ & $8-20$ & $200 \mathrm{k}-1 \mathrm{M}$ \\
\hline Fuel cells H2 & 350 & 700 & \\
\hline Fuel cells DMFC & $800-1200$ & 4400 & \\
\hline
\end{tabular}

A short description of the physical phenomena (presented below) used in EH shows their possibilities and limitations in relation to their potential use in the construction of $\mathrm{EH}$ generators.

\subsection{Phenomena using kinetic energy}

The kinetic energy is the most accessible form of energy, for both - human and environment - types of its source. Using any kind of movement or deformation of EH devices' components, we can convert them into electricity. In this group we distinguish three main phenomena: the piezoelectric, electrostatic, and electromagnetic (magnetic induction) ones. The first two phenomena can be used in the construction of micro-scale generators; the third one is used in generators with significantly larger overall dimensions [11, 21]. Analyzing the mechanical structures of EH generators, we can distinguish two dominant structures. The first one uses vibrations or movements of elements with an inertial mass, whereas the second - elastic deformation of structures that are potential generators of electricity (e.g. the piezoelectric effect).

\section{Piezoelectric generators}

The classical piezoelectric effect consists in polarization of a crystal in one direction caused by its mechanical deformation. And vice versa, an external electric field can deform a crystal. This phenomenon is explained by movement of ions in the crystal lattice that produces an internal electric field in the crystal. The higher the degree of symmetry of a crystal, the less clear the direction of its polarization. In crystals with a center of symmetry, this phenomenon does not occur. The polarization $P_{i}$ generated in a crystal under the influence of a mechanical stress $\sigma_{j k}$, is a linear function (2.1):

$$
P_{i}=d_{i j k}^{E} \cdot \sigma_{j k},
$$


where: $\sigma_{j k}$ - is a tensor of the second order, $d^{E}{ }_{i j k}-$ is a piezoelectric modulus (factor) of the crystal (measured at a fixed value of the electric field strength $E$ ) which specifies in a quantitative way its piezoelectric properties.

The piezoelectric modulus $d_{i j k}$ creates a tensor of the third order. They can also be written in the form of a matrix with the indexes $m$ and $n$. The equation (2.1) describes a simple piezoelectric effect. The same crystal, placed in an external electric field with the intensity $E_{i}$, is deformed by $\eta_{j k}$. This deformation is a linear function of the electric field components. The inverse piezoelectric effect is determined by $(2.2)$ :

$$
\eta_{j k}=d_{i j k}^{\sigma} \cdot E_{i}
$$

where: $d^{\sigma} \sigma_{i j k}$ - is the piezoelectric modulus (factor) of the crystal measured at a fixed value of the mechanical stress $\sigma_{j k}$.

When the direction of change of the polarization component $P_{i}$ in a simple piezoelectric phenomenon is perpendicular to the action of external mechanical stresses, then the observed phenomenon is known as the transverse piezoelectric effect. However, if the direction of change of polarization component $P_{i}$ is parallel to the direction of stress, then such a phenomenon is referred to as the longitudinal piezoelectric effect.

A typical piezoelectric generator is a plate (or a stack of plates) of a piezoelectric material on which the opposite surfaces form conductive plate electrodes. The resulting circuit has a structure similar to that of a capacitor, the dielectric being a piezoelectric material.

A significant feature of the longitudinal effect is that the total charge appearing on the electrodes depends only on the values of applied force and piezoelectric modulus, and does not depend on the geometrical dimensions of the piezoelectric plate. In the transverse effect, the total charge generated on the surface of electrodes is a function of its dimensions [13].

A typical generator circuit using the piezoelectric effect has the form of an elastic beam $(K$ - an elasticity constant) which is made of piezo ceramic (e.g. PZT), or is wrapped up in a foil of piezo material (e.g. PVDF) at the end of which the mass $M$ is fixed. For optimizing the energy efficiency of such a generator, its operation requires vibrations with a frequency close to the natural frequency of the elastic beam. This makes the design of the mechanical structure of the beam a challenge, especially if we are not able to predict accurately the kind of vibrations that our spring element will be subjected. Pulsation of the natural vibration, $\omega_{0}$, is a function of the elasticity constant $K$ and the mass $M$ :

$$
\omega_{0}=\sqrt{\frac{K}{M}}
$$

where: $K=\frac{3 E I}{L^{3}}, E-$ Young's modulus, $I-$ the moment of inertia, $L-$ the length of the beam.

This situation practically would require construction of a number of generators designed for a specific frequency each. A solution to this problem is a polydactylous system consisting of structures containing a series of elastic beams which differ in dimensions and material properties, e.g. the values of their elasticity constant or Young's modulus (Fig. 2.2), and thus they are characterized by several different natural frequencies [13, $21-23]$.
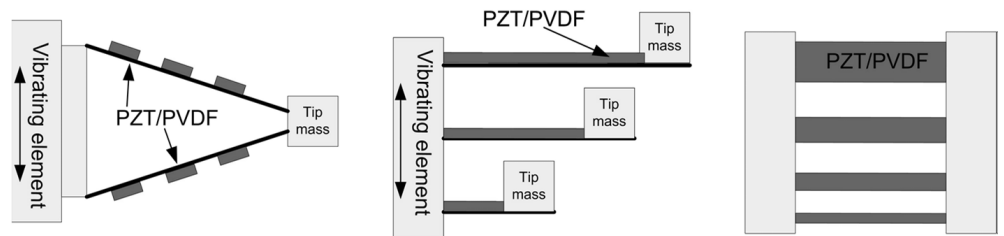

Fig. 2.2. The piezoelectric polydactylous generator. 
The result is a mechanically compact structure that adapts to the expected range of vibration frequencies.

\section{Electrostatic generators}

The basic principle of an electrostatic generator is movement of parts of the generator in the electric field. Such systems are common passive circuits that require an external power supply to initiate the conversion process. A typical structure of the electrostatic generator uses a flat capacitor, most often with air as a dielectric. Popular designs of flat capacitors made in the MEMS technology operate in two modes: the flat capacitor working with a constant charge and the comb capacitor working with a constant voltage $[10,12,24]$.

In the first case, the conversion cycle begins when the system reaches its maximum capacity $C_{\max }$. From then, the capacitor is charged by an external source. As a result of an external impact causing a change of the capacitor parameters (increasing the distance between the plates), the capacitor capacity decreases to its minimum. The electrical energy obtained during each complete cycle, can be described by $(2.4)[13,24]$ :

$$
E=\frac{1}{2} Q_{e}^{2}\left(\frac{1}{C_{\min }}-\frac{1}{C_{\max }}\right) .
$$

In the second case, with a constant voltage polarizing the capacitor, a change of its capacitance results in a change of the charge (the decrease of capacity causes a the decrease of charge stored in the capacitor). The conversion cycle begins when the capacitance reaches its maximum. The capacitor is polarized by a voltage $U_{z}$ from an external source. This voltage is kept constant during the entire conversion cycle. At a constant voltage, changing the capacitance according to a change of the distance between the plates induces a current flow in the external circuit of the capacitor. The total amount of energy that is obtained during one cycle is obtained from (2.5):

$$
E_{U_{z}}=U_{z}^{2}\left(C_{\max }-C_{\min }\right)
$$

The power of this type of systems varies from more than a dozen $\mu \mathrm{W}$ to $1000 \mu \mathrm{W}$, depending on the surface of electrodes and the polarization voltage [13].

The main problem in this type of systems is the need for external polarization voltages with relatively large values, often exceeding $100 \mathrm{~V}$. Independence of the generator from the need for an external power source may be possible with a system comprising electrets, instead of using an air capacitor $[11,13]$. This results in the direct conversion of the mechanical energy into the electrical energy without an additional external energy source. Electrets are dielectric materials with a quasi-fixed electrical polarity. One of their main advantages is the possibility of using them in micro-size devices in standard MST processes.

\section{Electromagnetic generators}

The kinetic energy is easily available in the natural environment. A source of this energy can be natural motion of humans, motor vehicles, or normal occurrences in any environment. Electromagnetic transducers are among the most efficient converters of the kinetic energy into electricity. The basis of their actions is the Faraday law of induction describing the relations between the induced electromotive force in a conductor, the magnetic field and the speed with which the conductor moves in this field.

$$
E=-\frac{\partial \Phi}{\partial t}=-\frac{d \Phi}{d x} \frac{d x}{d t}=-\frac{d \Phi}{d x} \vartheta
$$

where $\vartheta-$ the speed at which the conductor moves in the magnetic field.

After closing the electrical circuit with a load resistance, an electric current appears in the moving system. This current is the source of the magnetic field directed opposite to the field 
which is the source of this current. The interaction of two magnetic fields is the source of mechanical force counteracting the movement of the conductor. The conductor movement results in transforming the mechanical energy into the electrical energy.

Very often the electromagnetic force counteracting the movement of the conductor is defined as a function of the electromagnetic damping $(D)$ and the velocity of that conductor. [13]:

$$
F_{E M}=D \frac{d x}{d t}=D \cdot \vartheta
$$

Achieving the maximum energy efficiency for an electromagnetic generator requires maximizing the electromagnetic factor, $D$. It can be proved [9] that the value of $\mathrm{D}$ depends on the coil impedance, the load resistance at which the generating coil is working, and the gradient of the magnetic flux associated with the generating coil (2.8).

$$
D=\frac{1}{R_{\text {Lod }}+R_{c}+j \omega \omega_{c}}\left(\frac{d \Phi}{d x}\right)^{2},
$$

where: $R_{L o d}$ - the load resistance of the generating coil, $R c$ - the coil resistance, $L c$ - the inductance of the generating coil, $\Phi$ - the magnetic flux associated with the coil.

The magnetic flux associated with the coil depends largely on the quality of permanent magnets used in generators, and on the area and the number of windings of the generating coils. Due to a relatively low frequency of vibrations which are used (typically below $1 \mathrm{kHz}$ ), the coil impedance to a large extent consists of its resistance.

\section{Photovoltaic generators}

The photovoltaic effect consists in creating in a solid state the electromotive force caused by the electromagnetic radiation. This phenomenon describes creation of the electrical potential as a result of optical rays acting on a p-n junction (between $p$-type and n-type semiconductors) or on an m-s junction (between metal and semiconductor). In the case of a p-n junction, the phenomenon occurs as a result of absorption of the light energy, creating additional current carriers, i.e. electrons and holes. Subsequently, there occurs a process of spontaneous spreading the holes to the $\mathrm{p}$ area and the electrons to the $\mathrm{n}$ area that creates the electric potential at the junction between the areas. This phenomenon was discovered in 1839 by a French physicist Antoine Henri Becquerel. The potential can be also created by directing light on a uniform semiconductor. This type of phenomenon is known as the Dembera effect and arises from the difference between diffusion velocities of electrons and holes created from the surface area and diffused into the semiconductor.

The photovoltaic effect is widely used in industrial production of electricity. Photovoltaic farms can generate power of 5 to $30 \mathrm{MW}$ Of course, it does not mean that this effect cannot be used in powering individual sensor nodes. Commercially available amorphous silicon photovoltaic cells have a $11 \%$ conversion rate and a voltage of $700 \mathrm{mV}$. Assuming that a node needs $2 \mathrm{~V}$, the serial connection of three cells provides a sufficient power. A characteristic of this type of power supplies is a large, up to $90 \%$, daytime variation of their performance, requiring energy storage systems, such as supercapacitors or batteries. Most solar cells are made from silicon, germanium, or selenium. Over $85 \%$ of cells available on the market are made of silicon. We can distinguish the following cells:

- Monocrystalline cells - made from a single monocrystalline silicon crystal, have a high efficiency (approximately 18 to $22 \%$ ) and a relatively high price.

- Polycrystalline cells - made from crystallized silicon, have an efficiency of 14 to $18 \%$ and a reasonable price.

- Amorphous cells - made of amorphous and non-crystallized silicon, have a low efficiency of 6 to $10 \%$ and a low price. 
- CdTe cells - made of semiconductor cadmium telluride CdTe. Usually the whole module is composed of one cell. Their efficiency is 10 to $12 \%$ and they have a good price-to-power ratio.

- CIGS cells - made from a mixture of semiconductors such as copper, indium, gallium, and selenium. The modules are often constructed as single cells. Their efficiency is 12 to $14 \%$. They can be manufactured by industrial printing which is a cheap and efficient production method.

Table 3 shows the materials and structures of photovoltaic cells and indicates their efficiency $[8,9]$.

Table 3. The specification of materials and structures of photovoltaic cells.

\begin{tabular}{|c|c|c|c|}
\hline $\begin{array}{c}\text { The base } \\
\text { material of the } \\
\text { cell }\end{array}$ & Material form & Structure & $\begin{array}{c}\text { The highest } \\
\text { efficiency for } \\
\text { cells available } \\
\text { on the market } \\
{[\%]}\end{array}$ \\
\hline \multirow{4}{*}{ silicon } & crystalline & $\begin{array}{l}\text { Monocrystalline } \\
\text { polycrystalline }\end{array}$ & $\begin{array}{l}24,7 \\
20,3\end{array}$ \\
\hline & microcrystalline & thin-film & 11,7 \\
\hline & amorphous & thin-film & 10,3 \\
\hline & $\begin{array}{c}\text { combination of cells with monocrystalline silicon } \\
\text { and coated with amorphous silicon }\end{array}$ & hybrid & 21,8 \\
\hline \multirow{4}{*}{$\begin{array}{l}\text { semiconductor } \\
\text { compounds }\end{array}$} & \multirow{2}{*}{ halide } & CdTe & 16,5 \\
\hline & & CIS, CIGS & 19,5 \\
\hline & \multirow{2}{*}{$\begin{array}{l}\text { compounds of elements of III and V group } \\
\text { GaAs, InP, etc. }\end{array}$} & GaAs & 25,8 \\
\hline & & $\ln P$ & 21,9 \\
\hline
\end{tabular}

\section{Thermoelectric generators}

Thermoelectric generators are small. They have no moving parts, they are resistant to a mechanical damage, they are silent, which makes them ideal as electrical micro-sources in such applications as medicine. The basic principle of a thermoelectric generator is the Seebeck effect, which describes migration of mobile carriers of the electric charge in a material by subjecting the material to a non-zero temperature gradient.

Placing the semiconductor structure in a field with a non-zero temperature gradient causes migration of charges from the hotter end of the structure to the cooler end, which creates a potential difference between both ends. A typical material structure used in construction of a thermoelectric generator consists of n-type (containing free electrons) and p-type (containing free holes) materials. The resulting difference of potentials can be written as:

$$
U=\alpha \cdot \Delta T,
$$

where: $\alpha$ is the Seebeck coefficient.

The best thermoelectric materials are heavily doped semiconductors. A coefficient determining the quality of the material for use in construction of thermoelectric generators is called figure of merit, $z T$, which is defined by $(2.10)[9,25]$.

$$
z T=\frac{\alpha^{2} T}{\rho \kappa},
$$

where: $\alpha$ is the Seebeck coefficient, $T$ - the absolute temperature, $\rho$ - the electrical resistivity, $\kappa-$ the thermal conductivity of the material. 


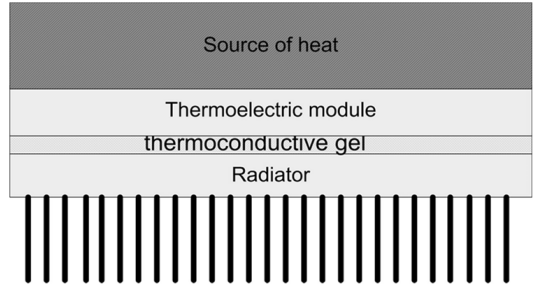

Fig. 2.3. An example of a thermoelectric generator structure.

A typical thermoelectric generator uses a plurality of n-type and p-type pairs that are connected electrically in series and thermally in parallel. Fig. 2.3 outlines the structure of a thermoelectric generator.

\section{Development of WSN solutions}

Foundations for sensor networks originated in military, defense and security areas of interest. The term "wireless sensor networks" eventually turned up and gained some attention [26, 27]. Popularity of WSNs skyrocketed after a survey presented by Akyldiz et al. [28]. WSNs evolved from labs and started to address real needs $[29,30]$. "Smart Dust" was coined as a concept of a node that should have a physical size of one cubic millimetre [31]. This goal still lies beyond the technical horizon in practical solutions. A more realistic approach to node construction will be discussed in the next subsection.

\subsection{Node hardware and physical layer}

The designs known as Mica, Mica2 and Mica2Dot that originated at Berkeley became the benchmark solutions that are still widely used despite their outdated architecture $[32,33]$. An important breakthrough occurred when the IEEE working group 802.15 created standards for lower layers of the communication protocol stack that employs a radio link as the physical medium [34, 35]. Most of transceivers on the market support one of these standards or their variants. They can be found in most solutions for WSN development $[36,37]$. Furthermore, newer nodes such as FireFly provide improved capabilities and make possible real-time operations [38]. The solutions matured and covered areas of medical devices, such as a sensor node named Eco that was designed at University of California to noninvasively monitor movement of limbs of pre-term infants [39]. A comprehensive list of motes dating from 1998 to 2010 can be found in [40].

The trend is to integrate a microcontroller (MCU) with other components, such as ADCs and transceivers. It leads to significant optimization of package size and energy consumption [31]. The architecture of a typical modern WSN node is presented in Fig. 3.1. Recent years have brought more commercially-oriented nodes such as Libelium Waspmote that can cover a wide range of applications like air and water pollution measurement, agriculture, surveillance, radiation, etc.

Wireless modules can be chosen to communicate with various protocols: ZigBee, WiFi, Bluetooth Low Energy, and some ISM bands. All major IC companies have some WSN solutions. Linear Technologies offers SmartMesh WirelessHART, which is a WSN solution designed mainly for industrial applications. It is built on LTC5800 chip and is advertised as a mote-on-chip with its integration of low power radio that was designed by Dust Networks. Atmel presented a new family of single-chip solutions based on 32-bit ARM Cortex-M0+ that is branded as SAM R21. Texas Instruments' CC430 is a family of system-on-chip components featuring low-power wireless communication that can be found on the panStamp NRG 2 
platform, which is well-suited for rapid development.

The devices and components presented above are just a few examples from a variety of solutions. WSNs came out of laboratories and successfully entered industry in the most recent decade. Nowadays we observe a rising demand for distributed measurement of different parameters, especially in the area of environmental monitoring. Available product portfolios span from single boards, through large WSN solutions, to IT cloud infrastructure. The next section presents practical applications of WSNs in environmental monitoring that employ energy harvesting.

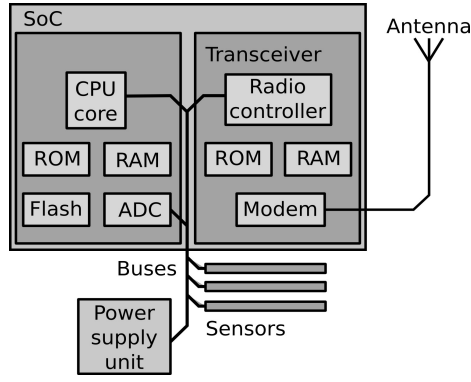

Fig. 3.1. A typical architecture of wireless sensor node.

\section{Energy Harvesting in real WSNs}

The authors focus on a review of EH techniques in systems developed for monitoring environmental parameters. Monitoring environmental parameters is a broad issue, so the authors divide WSNs into three groups which will be discussed in turn:

- The networks monitoring parameters of the air and atmosphere quality.

- The networks monitoring the quality of water and aquatic environment.

- The networks designed to monitor cultivated areas and agriculture.

In the review we present only physically-existing networks and measurement nodes. We do not analyze theoretical considerations related to the optimization of energy consumption by measurement nodes.

\subsection{Monitoring of air parameters}

Due to the increasing awareness of the health risks associated with air pollution [41], people and local governments are installing sensor networks to monitor the air quality. In these monitoring systems the measurement nodes are spread over vast areas. The most commonly monitored areas are those where pollutants might exceed permissible concentrations. These are mostly urban areas, main transport routes, and industrialized areas. It would seem that in the urban areas the problem of network power supply is easy to solve. There is, after all, an energy infrastructure that can be used to power the measurement nodes in monitoring networks. However, the legal restrictions concerning specific locations of measurement stations often make it impossible to use the energy infrastructure and the nodes must have their autonomous power supply units. A power supply of stations can be constructed using battery systems and supporting EH techniques. The easiest approach is to use the battery power. However, besides its simplicity such an approach offers an average node lifetime of only several months [42]. For the application presented in this paper, the lifetime of the node activity is acceptable, especially 
as the station is used to monitor the air quality indoors, where the battery replacement is straightforward. In situations when a measurement node operates outdoors, in a urban space, the battery replacement can be troublesome and, above all, expensive - considering a large number of stations and variety of locations. Significant lengthening of the node lifetime, and thus limiting the operating costs of the entire system, is obtained by using power supply systems supported by EH systems.

An example of a simple network monitoring only the air temperature is the system described in [43] where the node is based on an MSP430 circuit and an nRF24L01 transceiver. Both components are characterized by a low energy consumption. A digital thermometer DS18B20 is a sensor. The power and energy unit comprises photovoltaic cells and lithium-ion batteries managed by a dedicated CN3063 chip that supervises charging processes. The node measures just one parameter and the communication module operates within an area of 100 meter diameter. However, the applied EH system completely satisfies the node energy demand, which is very important.

Another example of using solar panels is the network presented in [44], where mobile nodes measure temperature and $\mathrm{CO}, \mathrm{CO}_{2}, \mathrm{SO}_{2}, \mathrm{NO}_{2}$, and $\mathrm{O}_{3}$ concentrations. A set of mobile nodes measure the air pollution in Lisbon (Portugal). As the node controllers PIC30F4013 devices are used. Communication takes place via GSM/GPRS, using a modem that needs an average of 390 $\mathrm{mW}$. The entire measurement station needs an average of $729 \mathrm{~mW}$. The authors use two 180 $\mathrm{mm}$ by $226 \mathrm{~mm}$ monocrystalline solar panels that power the station in good sunlight conditions. However, in the winter months the energy source is not sufficient and an additional panel has to be installed. The power supply system is buffered by a battery with a capacity of $2 \mathrm{Ah}$ and a rated voltage of $12 \mathrm{~V}$. In the above example, the problem of adequate energy efficiency is solved by increasing the number of photovoltaic cells. Such an approach is not technically possible to use everywhere, nevertheless in situations where one can use such a solution, it is a relatively cheap and efficient source of energy.

An interesting possibility using various EH techniques to power a measurement network node is described in $[45,46]$. The system is currently in the testing phase. It monitor nines parameters: $\mathrm{CO}, \mathrm{NO}, \mathrm{NO}_{2}, \mathrm{CL}_{2}, \mathrm{H}_{2} \mathrm{~S}$ and $\mathrm{NH}_{3}$ concentrations, pressure, temperature, and humidity. The measurement node is based on an PIC24 XLP microcontroller and a communication system operating in the $433 \mathrm{MHz}$ band from Linx Technology. The sensors and circuits that are conditioning and processing the measurement signal are based on CMOS circuits. This leads to one measurement cycle lasting 20 minutes and consuming energy of only $2.75 \mathrm{~J}$. The node power supply consists of a battery and four different systems of obtaining energy from the environment. In optimum operating conditions, the system is able to obtain energy of $137 \mathrm{~J}$ per day. Most of the energy $(125 \mathrm{~J}+4.3 \mathrm{~J})$ is obtained by two photovoltaic panels with dimensions of $96 \mathrm{~mm}$ by $72 \mathrm{~mm}$ and $96 \mathrm{~mm}$ by $48 \mathrm{~mm}$. The bigger panel is dedicated to acquire energy from sunlight, whereas the smaller one - from artificial light. The power supply contains 6 high-efficient TG12-2.5-01L thermoelectric generators, using the Peltier effect to produce daily $6.2 \mathrm{~J}$. A Powercast P211 circuit is a system which converts the electromagnetic field energy into electricity. In this case, the energy is acquired from the EH field in the $900 \mathrm{MHz}$ band. This circuit acquires approximately $1.8 \mathrm{~J}$ per day. Another element of $\mathrm{EH}$ is a circuit that converts the mechanical energy of vibrations into the electrical energy using a piezoelectric wafer made of PZT. In the described node the EH systems are able to secure the node operation (reducing the frequency measurement to two cycles per hour). The authors were able to create an energetically self-sufficient measurement node measuring 9 parameters. Essential to the EH in this example is the node location and the frequency of measurement, whereas the different techniques of obtaining energy make the node able to operate autonomously in all conditions. 


\subsection{Monitoring water parameters}

The aquatic environment is another area where the WSN capabilities are widely used. As in the case of monitoring the air quality, the purity and quality of water has an increasing importance for the economy and society. In this regard, there are systems that monitor the quality of inland water reservoirs [47], rivers [48], seas, and oceans [49]. Depending on the needs, either surface or abyssal parameters of water and aquatic environment are measured. Also, the measurement stations differ in complexity. There are stations weighing $1 \mathrm{~kg}$ that measure three parameters [50], as well as large measurement stations with a displacement of $400 \mathrm{~kg}$, measuring several parameters of the aquatic environment [51]. Regardless, the lifetime of a measurement node is important. To ensure the system efficiency, it should operate as long as possible. Since using the energy infrastructure is impossible (because it does not exist in this environment), the measurement stations are powered by battery systems. In these conditions, the EH systems are the indispensable parts of every station because without them the battery energy would be quickly exhausted.

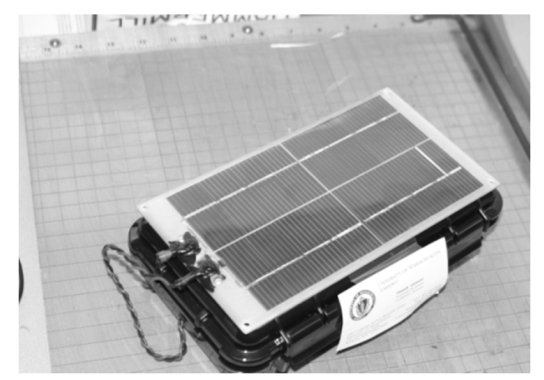

Fig. 4.1. A measurement node for monitoring water parameters (used with the permission from Prof. Prashant Shenoy).

Figure 4.1 shows a small measurement node of the network monitoring parameters of water in rivers [52]; it is based on an ARM Gumstix controller. It measures the basic parameters of water and sends the data via the IEEE 802.11 protocol stack. The supply block consists of a battery with a capacity of $6.1 \mathrm{Ah}$ and a nominal voltage of $3.7 \mathrm{~V}$. The battery provides with solar panel harvesting, in optimal lighting conditions, up to $700 \mathrm{~mW}$. A fully autonomous station that measures the parameters of water in the area of the Australian coral reef is presented in [53]. This station is designed to measure the water temperature and insolation conditions prevailing under the water surface. The station is also equipped with the humidity sensors that alarm a water leakage into the electronics. The station controller is an MICAz system. It is integrated with a ZigBee transceiver, a system energetically optimized for use in the WSN nodes. The supply block consists of two NiMH batteries, a solar panel, and a power management system. The task of the management circuit is adequate monitoring the battery and switching between the charging mode (collecting energy from the panel) and the discharging mode (service of measurement process). This approach enables to increase the efficiency and battery lifetime.

In the network that monitors the depth of rivers presented in [48], the node is based on an MSP 430 chip. The data transfer takes place via ZigBee. The measurement data are obtained from the GPS, a pressure sensor, and an ultrasound head measuring the distance from the surface to the bottom of the river. The power supply system consists of a solar panel and a set of 6 supercapacitors instead of an electrochemical battery. According to the authors of this research, this approach extends the system lifetime from 500 to 50000 cycles of full charge and discharge. 
The power supply circuits of the measurement network node can have different types of components for energy buffering. One example is a network used for monitoring nitrogen compounds in waters of rivers, shown in [54], where a supercapacitor is added to the battery system and the whole system is recharged by a photovoltaic panel.

The measuring-alarming station, shown in [55] and illustrated in Fig. 4.2, measures seven basic water parameters: temperature, $\mathrm{pH}$, turbidity, conductivity, and concentrations of: chloride, ammonia and hydrocarbons. It is based on a built-in MOXA computer and transmits the data in the $868 \mathrm{MHz}$ band on a distance of up to $5 \mathrm{~km}$. Such a large station takes an average of $12 \mathrm{~W}$. This in turn implies a comprehensive power system whose base is a lead-acid battery with a capacity of $110 \mathrm{Ah}$ at $12 \mathrm{~V}$ and an assisting power system consisting of five photovoltaic cells with a total area of $3.6 \mathrm{~m}^{2}$ and a fuel cell of $5 \mathrm{kWh}$, operated only in critical conditions.

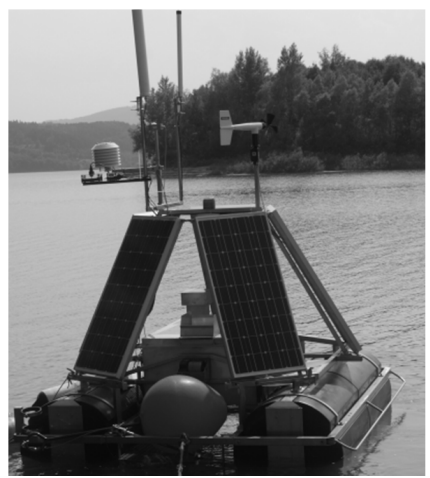

Fig. 4.2. A measurement station for monitoring the quality of drinking water.

There are also systems that combine different techniques of obtaining energy from a variety of buffering systems. The node [56] uses a supercapacitor and a battery system as energy storage elements, together with a piezoelectric resonator that converts the energy of deep sea waves into the electric energy, and a system of microbial fuel cells, where energy is obtained through the microbiological activity of bacteria on appropriate bio-degradable substrates.

The above-cited examples employ the same EH methods and ways to use them effectively, as in the case of air monitoring. The aim is to minimize the energy consumed by a station. The next step is to optimize the transmission channel, its length and the frequency of data transfer. We are also dealing with combining the EH methods and their proper management, and selecting the energy storage elements.

\subsection{Monitoring for farming and forestry}

The systems for monitoring environmental parameters, implemented to support agriculture and other sectors of cultivation, do not differ substantially from those presented above. The specificity of their work is mainly based on measurement of several important parameters and operating in the areas of cultivation. The most commonly monitored are large areas of cultivation. An example of such a network can be the network shown in [57], where the nodes measure parameters in a vineyard. A node monitors the temperature and humidity of both the air and the soil, as well as the pressure and insolation. The daily demand for energy of a node amounts to $330 \mathrm{~mW}$. The three-hour operation of a $70 \mathrm{~mm}$ by $55 \mathrm{~mm}$ solar panel can acquire $550 \mathrm{~mW}$, which makes the node fully autonomous. Another system monitoring the parameters of a vineyard is that presented in [58]. The system node measures the same set of parameters as the previous one, supplementing the data with measurements of the wind direction and rainfall 
intensity . The power supply system consists of a battery of $4.5 \mathrm{Ah}$ at $6 \mathrm{~V}$ and a $9 \mathrm{~V}$ solar panel. This node, however, does not work continuously. The basic unit of node operation is a cycle of 7 days after which it is necessary to transfer the data to the system gateway. Then, the node can be recharged and moved to another location.

An innovative approach to the problem of measurement node power is proposed in [59], where the node monitoring avocado plantations is powered by the energy gained from converting the electrical potential difference occurring in different parts of the plant into electricity. The node is currently being tested and the results can be considered very promising.

Besides plantation crops, forests can also be monitored . In Australia a system that contains 200 nodes, based on a dedicated CSIRO controller, measures the parameters of soil moisture, temperature, and the intensity of light and also the leaf wetness in a rainforest (Queensland) [60]. Each node is supported by a solar panel and can transmit data every five minutes. Fig. 4.3 shows a node in the rainforest. The system monitors the fire symptoms and the parameters of forest environment sensitive to their occurrence. An example of such a system is the network working in Sequoia National Park in the USA [61], where the measurement node is based on a dedicated WSN Tomte Sky controller with an embedded ZigBee module; it measures the temperature, humidity, and insolation. The power system has a lead acid battery with a capacity of $2.2 \mathrm{Ah}$ at $12 \mathrm{~V}$ and is supported by a solar cell. The system nodes were installed on trees and the cell efficiency assured the energy self-sufficiency of each node. A similar measurement task was carried out by the node presented in [62]. The main purpose of the system was quick detection of fire. The authors designed a miniature node measuring the air temperature and transmitting data using the ZigBee protocol on distances of up to $400 \mathrm{~m}$.

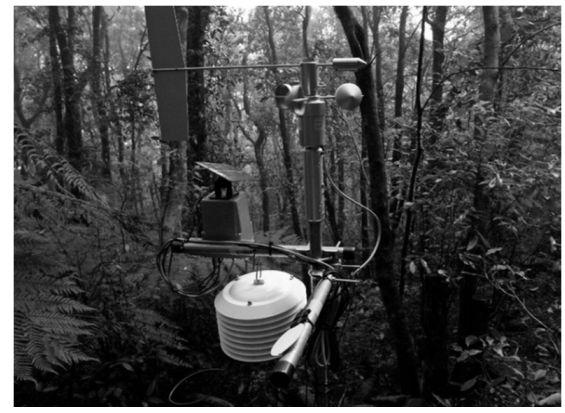

Fig. 4.3. A node for monitoring rainforest parameters (photo: CSIRO, CC BY 3.0).

The power block contains a micro windmill-structured turbine, which assures acquiring an average power of $3 \mathrm{~mW}$ at a wind speed of $3 \mathrm{~m} / \mathrm{s}$, and was independently developed by the authors. The network containing 400 measurement nodes was deployed in the Uwharrie forest in California To send a full set of data it needs energy supplied as a result of only $13 \mathrm{~s}$ of the turbine operation.

As in the previous applications, monitoring networks for agriculture can combine different methods of saving and generating energy to maintain a greater energy independence and longer operation of the nodes. An example is a node designed to monitor the air temperature and the soil humidity [63]. This node is based on an FPGA and digital sensors. The power supply system consists of a Li-Ion battery with a capacity of $6.4 \mathrm{Ah}$ at $3.7 \mathrm{~V}$, a photovoltaic panel, and a thermoelectric TEG panel. After implementing the author's algorithm for battery charging, the node batteries have been charged to the maximum level after 11 days during normal operation of the node.

Another example of a hybrid energy generation are the nodes of the network monitoring the environment of a vineyard [64]. The nodes are mobile stations measuring the temperature, 
insolation, humidity of air and soil. They are based on a dedicated WSN JN5121 u-controller with an integrated ZigBee module. The power system is larger and comprises three Ni-Ion batteries with a capacity of $650 \mathrm{mAh}$ at $1.5 \mathrm{~V}$, a cylindrical $60 \mathrm{~mm}$ by $200 \mathrm{~mm}$ wind turbine, a hydro-generator connected to the vineyard irrigation system, and an $0.5 \mathrm{~W}$ solar panel. After testing the network in the vineyard, it turned out that the energy supplied by the solar panel is sufficient to power the node. The energy gained from the wind generator and the hydrogenerator was significantly lower and its value was also difficult to predict. In the final version the authors did not use these generators, thereby reducing the cost and weight of the node and increasing its mobility.

\subsection{Open research}

Based on the above papers we conclude that energy harvesting is now an indispensable part of WSNs used in monitoring the environment. EH gives the opportunity for nodes to become independent of the infrastructure power. This is crucial in many measurement systems [65]. We can distinguish the systems by combinations of methods that extend the node operation capabilities:

- Optimizing hardware - the tendency of building nodes based on microcontrollers and energy-efficient communication modules adapted for the Internet of Things technology.

- Increasing the dimensions of EH - the size of an EH element is a major factor of its efficiency. In the case of monitoring the air parameters in the natural environment, increasing the node size is not problematic. The most commonly increased is the active surface of a photovoltaic panel.

- Combining multiple energy harvesting techniques - in this case the electricity is acquired simultaneously from multiple energy converters. This enables to operate the node independently of the current environment and weather conditions. This approach enables to obtain a greater average of energy for a given period of time (day, month), but is more expensive.

- Optimizing the data transmission involving the use of energy - efficient radio links dedicated to working in measurement systems.

\section{Conclusions}

First of all, we observe that making WSNs for environmental monitoring is still a challenging and complex task, despite advances in electronics, network algorithms, power efficiency, and energy storage. Broad knowledge from different areas of engineering is needed for a successful design of such systems.

This paper focuses on the hardware issues of WSN development and the authors had to carefully choose the included examples. We had to make hard decisions to skip descriptions of some motes or chips. Therefore, we kindly ask readers and hardware developers to forgive us this reduction as it was caused only by a limited size of the paper. We believe that we had pointed out quite a few interesting topics so that you could look for individual and interesting paths of research.

In the second part of the paper we showed that there is a set of technically feasible EH methods ready to choose from. However, no single, optimal method exists and there are no clear guidelines concerning satisfying selection of the most appropriate one. Each situation requires from the engineers and system designers to pick one or a few energy harvesting methods based on the system and location limitations. For example, the piezoelectric methods are very efficient methods provided that mechanical vibrations are close to their resonant frequency. The electromagnetic generators are very efficient, but they require inconvenient magnets and large 
coils. The wind or water turbines are practical examples of such devices. The photovoltaic methods are quite effective, especially when perovskite materials are used, but are heavily dependent on light conditions which are usually determined by the weather. The thermoelectric methods, such as in Peltier modules, are quite interesting but only when there is a significant gradient of temperature available. Eventually, combinations of different methods are sometimes tested but such an approach complicates design and increases the system costs.

In the third section we focused on the origins and development of WSN hardware. We revised the division of a broad spectrum of WSN development problems into smaller areas of research. We recounted milestones in evolution of the node hardware, such as Mica and TelosB platform families. The hardware that stands behind these brand names was reviewed. Then, we focused on newer chips that had been introduced to the market within the last five years. It can be stated that WSNs evolved from a scientific curiosity into mature commercial products within about three decades.

The last part of this paper contains a survey of carefully chosen examples of WSNs that were used in environmental monitoring and employed one or more methods of energy harvesting. We showed the practical advantages obtained in some projects applying a combination of several methods Also, we showed the challenges that are brought by such an approach. Different solutions for monitoring the air and water quality, detecting forest fire symptoms and supervising agricultural zones were thoroughly discussed.

On a regular basis, interest in distributed measurements takes on new shapes, such as represented by new ideas like the Internet of Things, smart cities, or cloud computing [66]. It is hard to tell where the progress will lead us, but the basic core of operations is still a challenging field of research and seems to stay the same: to accurately measure specific parameters, effectively transmit data through the wireless network, and reliably receive them in a remote location.

To sum up this paper, we would like to state that creating measurement systems in the form of WSNs is a demanding but very rewarding process. We are grateful that we had this opportunity to share our humble experience gained from this exciting area of research.

\section{References}

[1] Pallikonda Rajasekaran, M., Radhakrishnan, S., Subbaraj, P. (2010). Sensor grid applications in patient monitoring. Future Generation Computer Systems, 26(4), 569-575.

[2] Sung, W.T., Chang, K.Y. (2014). Health parameter monitoring via a novel wireless system. Applied Soft Computin, 22, 667-680.

[3] Bhuiyan, M.Z.A., Wang, G., Cao, J., Wu, J. (2015). Deploying Wireless Sensor Networks with FaultTolerance for Structural Health Monitoring. IEEE Transactions on Computers, 64(2), 382-395.

[4] Sheng, Z., Mahapatra, C., Zhu, C, Leung, V.C.M. (2015). Recent Advances in Industrial Wireless Sensor Networks Toward Efficient Management in IoT. IEEE Access, 3,622-637.

[5] Barac, F., Gidlund, M., Zhang, T. (2014). Scrutinizing Bit- and Symbol-Errors of IEEE 802.15.4 Communication in Industrial Environments. IEEE Transactions on Instrumentation and Measurement, 63(7), 1783-1794.

[6] Muñoz-Gea, J.P., Manzanares-Lopez, P., Malgosa-Sanahuja, J., Garcia-Haro, J. (2013). Design and implementation of a P2P communication infrastructure for WSN-based vehicular traffic control applications. Journal of Systems Architecture, 59(10) Part B, 923-930.

[7] Markevicius, V., Navikas, D., Zilys, M., Andriukaitis, D., Valinevicius, A., Cepenas, M. (2016). Dynamic Vehicle Detection via the Use of Magnetic Field Sensors. Sensors, 16(1), 78.

[8] Winkler, M., Tuchs, K. D., Hughes, K., Barclay, G. (2008). Theoretical and practical aspects of military wireless sensor networks. Journal of Telecommunications and Information Technology, 2, 37-45.

[9] Prima, S., Inman, D. (2009). Energy Harvesting Technologies. New York: Springer 2009. 
[10] Mitcheson, P., Yeatman, E., Kondala Rao, G., Holms, A., Green, T. (2008). Energy harvesting from human and machine motion for wireless electronic devices. Proc. of the IEEE, 96(9), 1457-1486.

[11] Lee, C., Lim, Y.M., Yang, B., at el. (2009). Theoretical comparison of the energy harvesting capability among various electrostatic mechanisms from structure aspect. Sensors and Actuators A Physical, 156(1), 208-216.

[12] Loreto, M., Moll, F. (2015). Review of Energy Harvesting Techniques and Applications for Microelectronics, Drayson Technologies.

[13] Wan, Z.G., Tan, Y.K., Yuen, C. (2011). Review on energy harvesting and energy management for sustainable wireless sensor networks. Proc. of the IEEE International Conference on Communication Technology (ICC $\left.T^{\prime} 11\right), 317-362$.

[14] Tan, Y.K., Panda, S.K. (2011). Self-autonomous wireless sensor nodes with wind energy harvesting for remote sensing of wind-driven wildfire spread. IEEE Transactions on Instrumentation and Measurement, 60(4), 1367-1377.

[15] Tuna, G., Gungor, V.C. (2016). Ch2 - Energy harvesting and battery technologies for powering wireless sensor networks. Industrial Wireless Sensor Networks, 25-38.

[16] Boisseau, S., Despesse, G., Seddik, B.A. (2012). Ch5. Electrostatic Conversion for Vibration Energy Harvesting. Small-Scale Energy Harvesting, Intech.

[17] Ren, X., Zelenay, P., Thomas, S., Davey, J., Gottesfeld, S. (2000). Recent advances in direct methanol fuel cells at Los Alamos National Laboratory. Journal of Power Sources, 86, 111-116.

[18] Gurau, B., Smotkin, E.S. (2002). Methanol crossover in direct methanol fuel cells: a link between power and energy density. Journal of Power Sources, 112, 339-352.

[19] Application Notes and FAQ, http://www.optixtal.com/applicationnotes.htm (Mar. 2016).

[20] The High-power Lithium-ion, http://batteryuniversity.com/learn/article/the_high_power_lithium_ion (Mar. 2016).

[21] Bhatnagar, V., Owende, P. (2015). Energy Harvesting for assistive and mobile applications. Energy Science \& Engineering, 3(3), 153-217.

[22] Calio, R., Bhaskar Rongala, U., Camboni, D., Milazzo, M., Stefanini, C., De Petris, G., Oddo, C.M. ( March 2014). Piezoelectric Energy harvesting Solutions. Sensors, 14(3), 4755 - 4790.

[23] Soo Kim, H., Kim, J., (2011). A review of Pizoelectric Energy Harvesting based on Vibration. International Journal of Precision Engineering and Manufacturing, 12(6), 1129-1141.

[24] Sheu, G.J., Yang, S.M., Lee, T. (2011). Development of a low frequency electrostatic comb-drive energy harvester compatiblie to SoC design by CMOS process. Sensors and Actuators A Physical, 1667(11),70-76.

[25] Vullers, R.J.M., Van Schaijk, R., Doms, I., Van Hoof, C., Mertens, R. (2009). Micropawer energy harvesting. Solid-State Electronics, 53, 684-693.

[26] Chong, C.Y., Kumar, S.P. (2003). Sensor networks: evolution, opportunities, and challenges. Proc. of the IEEE, 91(8), 1247-1256.

[27] Swanson, D.C., Hall, D.L. (1994). Real-time data fusion processing of internetted acoustic sensors for tactical applications. Multisensor Fusion and Integration for Intelligent Systems. IEEE International Conference on MFI'94, 443-446.

[28] Akyildiz, I.F., Su, W., Sankarasubramaniam, Y., Cayirci, E. (2020). A survey on sensor networks. IEEE Communications Magazine, 40(8),102-114.

[29] Yick, J., Mukherjee, B., Ghosal, D. (2008). Wireless sensor network survey. Computer Networks, 52(12), 2292-2330.

[30] Dziadak, B., Makowski, L., Michalski, A. (2011). Embedding wireless water monitoring system in Internet. Przeglad Elektrotechniczny, 87(4), 246-248.

[31] Calhoun, B.H., Daly, D.C., Verma, N., Finchelstein, D.F., Wentzloff, D.D., Wang, A., Cho, S., Chandrakasan, A.P. (2005). Design considerations for ultra-low energy wireless microsensor nodes. IEEE Transactions on Computers, 54(6),727-740.

[32] Hill, J.L. Culler, D.E. (2002). Mica: a wireless platform for deeply embedded networks. IEEE Micro, 22(6), 12-24. 
[33] Pham, C. (2014). Communication performances of IEEE 802.15.4 wireless sensor motes for data-intensive applications: A comparison of WaspMote, Arduino MEGA, TelosB, MicaZ and iMote2 for image surveillance. Journal of Network and Computer Applications, 46, 48-59.

[34] IEEE Standard for Information technology - Local and metropolitan area networks - Specific requirements - Part 15.1 a: Wireless Medium Access Control (MAC) and Physical Layer (PHY) specifications for Wireless Personal Area Networks (WPAN). IEEE Std 802.15.1-2005 (Revision of IEEE Std 802.15.1-2002), 2005, $1-700$.

[35] IEEE Standard for Local and metropolitan area networks - Part 15.4: Low-Rate Wireless Personal Area Networks (LR-WPANs). IEEE Std 802.15.4-2011 (Revision of IEEE Std 802.15.4-2006), 2011, 1-314.

[36] Chu, H., Xie, Z., Shao, Y., Liu, Q., Mi, Z. (2010). Design and implement of WSN based on Bluetooth and embedded system. 2010 International Conference on Computer Application and System Modeling (ICCASM), 5, V5-641-V5-644.

[37] Yu, C., Cui, Y., Zhang, L., Yang, S. (2009). ZigBee Wireless Sensor Network in Environmental Monitoring Applications. 5th International Conference on Wireless Communications, Networking and Mobile Computing, WiCom, 1-5.

[38] Buevich, M., Rajagopal, N., Rowe, A. (2013). Hardware Assisted Clock Synchronization for Real-Time Sensor Networks. Real-Time Systems Symposium (RTSS), 2013 IEEE 34th, 268-277.

[39] Park, C., Liu, J., Chou, P.H. (2005). Eco: an Ultra-Compact Low-Power Wireless Sensor Node for Real-Time Motion Monitoring. Proc. of The Fourth International Conference on Information Processing in Sensor Networks, 398-403.

[40] Webster, J.G., Eren, H. (2014). Measurement, Instrumentation, and Sensors Handbook, Second Edition. CRC Press.

[41] Adamkiewicz, Ł., Badyda, A.J., Gayer, A., Mucha, D. (2015). Disability-adjusted life years in the assessment of health effects of traffic-related air pollution. Advances in Experimental Medicine and Biology, 834, pp. $15-20$

[42] Jelicic, V., Magno, M., Brunelli, D., Paci, G., Benini, L. (2013). Context-Adaptive Multimodal Wireless Sensor Network for Energy-Efficient Gas Monitoring. IEEE Sensors Journal, 13, 328-338.

[43] Yi, G., Guiling, S., Weixiang, L., Yong, P. (2009). Wireless sensor node design based on solar energy supply. 2009 2nd International Conference on Power Electronics and Intelligent Transportation System (PEITS), 203-207.

[44] Carvalho, V., Lopes, J.G., Ramos, H.G., Correa Alegria, F. (2009). City-wide mobile air quality measurement system. 2009 IEEE Sensors, 546-551.

[45] Touati, F., Galli, A., Crescini, D., Crescini, P., Mnaouer, A. (2015). Feasibility of air quality monitoring systems based on environmental energy harvesting. 2015 IEEE International Instrumentation and Measurement Technology Conference (I2MTC), 266-271.

[46] Touati, F., Legena, C., Galli, A., Crescini, D., Crescini, P., Mnaouer, A.B. Environmentally Powered Multiparametric Wireless Sensor Node for Air Quality Diagnostic. Sensors and Materials, 27(2), 177-189.

[47] Michalski, A., Staroszczyk, Z., Dziadak, B. (2009). Selected aspects of wireless sensor network for petroleum derivative monitoring system. Przeglad Elektrotechniczny, 85(4), 1-3.

[48] Hormann, L.B., Glatz, P.M., Steger, C., Weiss, R. (2010). A wireless sensor node for river monitoring using MSP430 and energy harvesting. 2010 4th European. Presented at the Education and Research Conference (EDERC), 140-144.

[49] Alippi, C., Camplani, R., Galperti, C., Roveri, M. (2008). Effective design of WSNs: From the lab to the real world. 3rd International Conference on Sensing Technology, 2008, ICST 2008, 1-9.

[50] Mihajlovic, Z., Joza, A., Milosavljevic, V., Rajs, V., Zivanov, M. (2015). Energy harvesting wireless sens or node for monitoring of surface water. 2015 21st International Conference on Automation and Computing (ICAC), 1-6.

[51] Dziadak, B., Makowski, L., Michalski. (2013). Some Practical Problems of Communications Reliability in Enviromental Monitoring Systems. Metrol. Meas. Syst., 20(3), 337-350.

[52] Shenoy, P., Ganesan, D., Irwin, D., Gummeson, J., Sharma, N., Somerville. T., Rivernet, A. (2016). Wireless 
Sensor Network for remote monitoring of riverbed ecosystems, http://sensors.cs.umass.edu/projects/ rivernet/

[53] Alippi, C., Camplani, R., Galperti, C., Roveri, M. (2011). A Robust, Adaptive, Solar-Powered WSN Framework for Aquatic Environmental Monitoring. IEEE Sensors Journal, 11, 45-55.

[54] Capella, J.V., Bonastre, A., Ors, R., Peris, M. (2013). In line river monitoring of nitrate concentration by means of a Wireless Sensor Network with energy harvesting. Sensors and Actuators B: Chemical, 177, 419427.

[55] Michalski, A., Dziadak, B., Makowski, L. (2008). Mobile-Observation-Point-Selected Aspects in Design and Signal Transmission. IEEE Transactions on Instrumentation and Measurement, 57, 1695-1702.

[56] Srujana, B.S., Neha, Mathews, P., Harigovindan, V.P. (2015). Multi-source Energy Harvesting System for Underwater Wireless Sensor Networks. Proc. of the International Conference on Information and Communication Technologies, ICICT 2014, Kochi, India, 46, 1041-1048.

[57] Rodriguez de la Concepcion, A., Stefanelli, R., Trinchero, D. (2014). A wireless sensor network platform optimized for assisted sustainable agriculture. 2014 IEEE Global Humanitarian Technology Conference (GHTC), 159-165.

[58] Matese, A., Di Gennaro, S.F., Zaldei, A., Genesio, L., Vaccari, F.P. (2009). A wireless sensor network for precision viticulture: The NAV system. Computers and Electronics in Agriculture, 69, 51-58.

[59] Konstantopoulos, C., Koutroulis, E., Mitianoudis, N., Bletsas, A., (2016). Converting a Plant to a Battery and Wireless Sensor with Scatter Radio and Ultra-Low Cost. IEEE Transactions on Instrumentation and Measurement, 65, 388-398.

[60] Gedda, R. (2009). 200 solar-powered network sensors to monitor rainforest. Techworld Australia. http://www.techworld.com.au/article/307279/200_solar-powered_network_sensors_monitor_rainforest/

[61] Burgess, S.S.O., Kranz, M.L., Turner, N.E., Cardell-Oliver, R., Dawson, T.E. (2010). Harnessing wireless sensor technologies to advance forest ecology and agricultural research. Agricultural and Forest Meteorology, 150, 30-37.

[62] Wu, X., Lee, D.W. (2014). An electromagnetic energy harvesting device based on high efficiency windmill structure for wireless forest fire monitoring application. Sensors and Actuators A: Physical, 219, 73-79.

[63] Philipp, F., Zhao, P., Samman, F.A., Glesner, M., Dassanayake, K.B., Maheswararajah, S., Halgamuge, S. (2012). Adaptive wireless sensor networks powered by hybrid energy harvesting for environmental monitoring. 2012 IEEE 6th International Conference on Information and Automation for Sustainability (ICIAfS), 285-289.

[64] Morais, R., Fernandes, M.A., Matos, S.G., Serôdio, C., Ferreira, P.J.S.G., Reis, M.J.C.S. (July 2008). A ZigBee multi-powered wireless acquisition device for remote sensing applications in precision viticulture. Computers and Electronics in Agriculture, 62, 94-106.

[65] Dziadak, B., Michalski, A. (2011). Evaluation of the Hardware for a Mobile Measurement Station. IEEE Transactions on Industrial Electronics, 58, 2627-2635.

[66] Lee, E.A., Rabaey, J., Hartmann, B., Kubiatowicz, J., Pister, K., Sangiovanni-Vincentelli, A., Seshia, S.A., Wawrzynek, J., Wessel, D., Rosing, T.S., Blaauw, D., Dutta, P., Fu, K., Guestrin, C., Taskar, B., Jafari, R., Jones, D., Kumar, V., Mangharam, R., Pappas, G.J., Murray, R.M., Rowe, A. (2014). The Swarm at the Edge of the Cloud. Design \& Test, IEEE. 31(3), 8-20. 